\title{
RICH SETS
}

\author{
C. T. CHONG
}

\begin{abstract}
Let $(V=L)_{\alpha}$ say that every bounded subset of $\alpha$ is an element of $L_{\alpha}$. We show that if $(V=L)_{\alpha}$, then every $X \subseteq \alpha$ of order-type $\alpha$ is rich, in the sense that every $\alpha$-degree above that of $X$ is represented by a subset of $X$.
\end{abstract}

Let $\alpha$ be an admissible ordinal. Following Jockusch [1] and Simpson [3], call a set $X \subseteq \alpha$ rich if every $\alpha$-degree above that of $X$ is represented by a subset of $X$. Soare [4] produced a set $X \subseteq \omega$ with no subsets of higher Turing degree ( $\omega$-degree), and Simpson [3] showed that every $\omega$-infinite nonrich set occupies a rather high Turing degree-above those of hyperarithmetical sets. We show in this paper that assuming a version of Gödel's Axiom of Constructibility $V=L$, there are many $\alpha$ 's where every $X \subseteq \alpha$ of order type $\alpha$ is rich.

Let $(V=L)_{\alpha}$ denote the following statement. Every $K \subseteq \alpha$ bounded below $\alpha$ is an element of $L_{\alpha}$ (called $\alpha$-finite). It can be seen that this is equivalent to saying that $\alpha$ is a cardinal and $L_{\alpha}=H_{\alpha}$. Under this assumption, one has in particular that every bounded $\omega$-sequence is $\alpha$-finite. The following discussion then makes sense under $(V=L)_{\alpha}$. Let $C$ be a subset of $\alpha$ and let $[C]^{\omega}$ denote the collection 'of $\alpha$-bounded subsets of $C$ of order type $\omega$. For each $\sigma<\alpha$ of cofinality $\omega$, choose an $\alpha$-finite increasing sequence $\left\{s_{\sigma, n}\right\}_{n<\omega}$ such that $\lim _{n \rightarrow \omega} s_{\sigma, n}=\sigma$. As there exists an $\alpha$-recursive well-ordering of $L_{\alpha}$, the map $\sigma \mapsto\left\{s_{\sigma, n}\right\}_{n<\omega}$ can be made partial $\alpha$-recursive. Let $P \subseteq[\alpha]^{\omega}$ be the $\alpha$-recursive set consisting of all increasing $\omega$ sequences $\left\{x_{n}\right\}_{n<\omega}$ such that if $\sigma=\lim _{n \rightarrow \omega} x_{n}$, then $x_{n}>s_{\sigma, n}$ for all $n$. Call $C \subseteq \alpha$ homogeneous for $P$ if $C$ is of order type $\alpha$ and $[C]^{\omega} \subseteq P$ or $[C]^{\omega} \cap P=\varnothing$.

LEMMA $\left((V=L)_{\alpha}\right)$. There is no set $C$ homogeneous for $P$.

Proof. Let $C \subseteq \alpha$ be homogeneous for $P$. Then clearly $[C]^{\omega} \subseteq P$, since if $\left\{c_{n}\right\}_{n<\omega}$ is any increasing $\omega$-sequence in $[C]^{\omega}$ with limit $\sigma$, there is always a subsequence $\left\{c_{n}^{\prime}\right\}_{n<\omega}$ of $\left\{c_{n}\right\}_{n<\omega}$ such that $c_{n}^{\prime}>s_{\sigma, n}$ for all $n$. Then $\left\{c_{n}^{\prime}\right\}_{n<\omega}$ is an element of $[C]^{\omega} \cap P$. On the other hand, choose two increasing sequences $\left\{x_{n}\right\}_{n<\omega}$ and $\left\{y_{m}\right\}_{m<\omega}$ in $[C]^{\omega}$ such that $x_{n}<y_{m}$ for all $m, n$. Write $\sigma=\lim _{n \rightarrow \omega} x_{n}$ and $\tau=\lim _{m \rightarrow \omega} y_{m}$. Then as $[C]^{\omega} \subseteq P$ we have $x_{n}>s_{\sigma, n}$ and $y_{m}>s_{\tau, m}$ for all $n, m$. As $C$ is of order type $\alpha$, it is possible to pick $x_{n}$ 's and $y_{m}$ 's so that $\sigma<\tau$. There is then an $m_{0}$ such that $s_{\tau, m_{0}}>x_{n}$ for all $n$. Now let $\left\{z_{j}\right\}_{j<\omega}$ be in $[C]^{\omega}$ such that $z_{j}=x_{j}$ for all $j \leqslant m_{0}$ and $z_{j}=y_{j}$ otherwise. Then $\lim _{j \rightarrow \omega} z_{j}=\tau$ and $\left\{z_{j}\right\}_{j<\omega}$ is not in $P$, contradicting the homogeneity of $C$.

Received by the editors July 27, 1979.

AMS (MOS) subject classifications (1970). Primary 02F27; Secondary 02K05.

$K e y$ words and phrases. Rich sets, $\alpha$-recursive, $\alpha$-degree, $\alpha$-finite, homogeneous. 
REMARK. The above argument actually shows that there is no homogeneous set of order type $\omega \cdot 2$.

TheOrem $\left((V=L)_{\alpha}\right)$. If $A$ is of order type $\alpha$, then $A$ is rich.

Proof. Let $\boldsymbol{P}$ be as defined above. By Lemma 1.1, $\boldsymbol{A}$ is not homogeneous for $\boldsymbol{P}$. For each $v<\alpha$, pick two increasing sequences $\left\{x_{n}^{v}\right\}_{n<\omega}\left\{y_{m}^{v}\right\}_{m<\omega}$ satisfying the following.

(i) $\lim _{n \rightarrow \omega} x_{n}^{v}<y_{0}^{v}$ and $\left\{x_{n}^{v}\right\} \in P,\left\{y_{m}^{\nu}\right\} \notin P$,

(ii) $\lim _{m \rightarrow \omega} y_{m}^{\mu}<x_{0}^{\nu}$ for all $\mu<v$.

From the proof of the Lemma, it can be seen that these sequences can be picked $\alpha$-recursively in $A$. Indeed at every stage $v$, if $x_{n}^{\mu}, y_{m}^{\mu}$ are all less than $z$ (for all $\mu<v$ ), then the ordinals $x_{n}^{v}$ and $y_{m}^{v}$ can be selected from among the first $\omega \cdot 3$ elements of $A \cap\{u \mid z \leqslant u<\alpha\}$. As $A$ is of order type $\alpha$, the process of selection of sequences will never end at any stage before $\alpha$.

Now let $B>_{\alpha} A$. We will construct $C$, a subset of $A$, such that $B$ and $C$ have the same degree.

Stage 0. Let $C^{0}=\varnothing$.

Stage $v>0$. Let $C^{v}=\left\{x_{n}^{v}\right\}$ if $v \in B$ and let $C^{v}=\left\{y_{m}^{v}\right\}$ if $v \notin B$.

Set $C=\cup C^{v}, v<\alpha$.

Clearly $C$ is a subset of $A$. We claim that $B$ and $C$ have the same degree. Take an $\alpha$-finite set $K$. Let $v$ be the least ordinal $\eta \operatorname{such}$ that $\sup \{z \mid z \in K\}<\min C^{\eta}$. Then $K \subseteq C$ if and only if $K \subseteq \cup C^{\mu}, \mu \leqslant v$. Now use $B$ to decide whether $\mu$ is in $B$ for each $\mu \leqslant v$. For each decision thus made, use $A$ to determine elements of $C^{\mu}$. Then $A \oplus B$ together provide information about $K$. But $A<_{\alpha} B$, and therefore $C<_{\alpha} B$. Now fix $v<\alpha$. To decide whether $v$ is in $B$, it is sufficient to know whether $C^{v}$ is in $P$. As $P$ is $\alpha$-recursive, it is enough to know that $C^{v}$ is. But $C^{v}$ is precisely the $v$ th $\omega$-sequence in the natural ordering of elements of $C$. It follows that $B \leqslant_{\alpha} C$. Thus $B$ and $C$ have the same degree and thus $A$ is rich.

The above result no longer holds in general if we allow $A$ to be of order type less than $\alpha$. As an example, take a singular cardinal $\alpha$ of cofinality $\kappa$. Let $A \subseteq \alpha$ be of order type $\kappa$. If $C \subseteq A$, the set $K=\{\nu \mid \nu<\kappa$ and the $\nu$ th element of $A$ is in $C\}$ is bounded below $\alpha$ and hence $\alpha$-finite (under $V=L$ ). Clearly $C<_{\alpha} A$ via the parameter set $K$. Furthermore, there are sets $A$ all of whose $\alpha$-infinite sets are of the same $\alpha$-degree. Thus let $f: \kappa \rightarrow \alpha$ be a strictly increasing cofinality function. Fix an $X \geqslant_{\alpha} f$. Let $A=\left\{\sigma(\nu) \mid X \cap f(\nu)=K_{\sigma(\nu)}\right\}$, where $\left\{K_{\rho}\right\}_{\rho<\alpha}$ is an $\alpha$-recursive enumeration of $\alpha$-finite sets and $\sigma(\nu)$ is the least $\rho$ such that $X \cap f(\nu)=K_{\rho}$. It is easy to check that $A \equiv_{\alpha} X$. Now $A$ is of order type $\kappa$ and therefore, by the above, every subset of $A$ is $\alpha$-recursive in $A$. On the other hand, let $C$ be an $\alpha$-infinite subset of $A$. Then $X<_{\alpha} C$ and so $A<_{\alpha} C$. (R. A. Shore informed us that J. Stillwell in his thesis [5] had observed this phenomenon when $\alpha=\aleph_{\omega}$ and $A=\left\{\boldsymbol{N}_{n} \mid n<\omega\right\}$.)

REMARK. Soare [4] used a weak version of the partition relation $\omega \rightarrow(\omega)^{\omega}$ to prove the existence of a set with no subsets of higher Turing degree. As even weak versions of infinite partition relations of the type $\alpha \rightarrow(\alpha)^{\alpha}$ contradicts the Axiom of Choice (AC) for $\alpha>\omega$ (cf. Kleinberg [2]), it will be interesting to see if Soare's 
result holds under an assumption consistent with AC. On the other hand, Martin has shown that the Axiom of Determinacy (AD) implies $\aleph_{1} \rightarrow\left(\aleph_{1}\right)^{\kappa_{1}}$, so it would appear that perhaps, assuming $A D$, Soare's result could be lifted to $\aleph_{1}$. But the use of $x_{1}$-choice, known to contradict $x_{1} \rightarrow\left(k_{1}\right)^{k_{1}}$, seems to be necessary. The possibility of such a generalization therefore remains uncertain.

Acknowledgement. We wish to express our thanks to S. Friedman and R. A. Shore for the comments they made on the original version of this paper, which led to the present revision.

\section{REFERENCES}

1. C. Jockusch, Upward closure and cohesive degrees, Israel J. Math. 15 (1973), 332-335.

2. E. M. Kleinberg, Infinitary combinatorics and the axiom of determinacy, Lecture Notes in Math., vol. 612, Springer-Verlag, Berlin and New York, 1977.

3. S. Simpson, Sets which do not have subsets of every higher degree, J. Symbolic Logic 43 (1978), 135-138.

4. R. I. Soare, Sets with no subset of higher degree, J. Symbolic Logic 34 (1969), 53-56.

5. J. Stillwell, Reducibility in generalized recursion theory, Ph. D. thesis, M.I.T., 1970.

Department of Mathematics, University of Singapore, Singapore 1025, Republic of Singapore 